Published in final edited form as:

Int Urogynecol J. 2016 November ; 27(11): 1705-1711. doi:10.1007/s00192-016-3022-8.

\title{
Mindfulness-Based Stress Reduction as a Novel Treatment for Interstitial Cystitis/Bladder Pain Syndrome: A Randomized Controlled Trial
}

\author{
Gregory Kanter, MD, MS, Yuko M Komesu, MD, Fares Qaedan, PhD, Peter C Jeppson, MD, \\ Gena C Dunivan, MD, Sara B Cichowski, MD, and Rebecca G Rogers, MD \\ University of New Mexico
}

\begin{abstract}
Purpose-Mindfulness-based stress reduction (MBSR) is a standardized meditation program that may be an effective therapy for Interstitial Cystitis/Bladder Pain Syndrome (IC/BPS), a condition exacerbated by stress. The aims of this study were to explore 1) whether MBSR improved IC/BPS symptoms 2) MBSR's feasibility/acceptability among women with IC/BPS.
\end{abstract}

Materials and Methods-This randomized controlled trial included women with IC/BPS undergoing $1^{\text {st }}$-or $2^{\text {nd }}$ line therapies. Women were randomized to continuation of usual care (UC) or an 8-week MBSR class + usual care (MBSR). Participants completed baseline and 8 week posttreatment questionnaires including the O'Leary-Sant Symptom Problem Index (OSPI), visual analog pain scale (VAS), Short Form Health Survey (SF-12), Female Sexual Function Index (FSFI), and Pain Self-Efficacy Questionnaire (PSEQ). The Global Response Assessment (GRA) was completed post-treatment. Analyses were performed using Student's t-test, Chi-square and MANOVA where appropriate.

Results-Eleven women randomized to UC and 9 to MBSR, without differences in group characteristics. More MBSR participants' symptoms were improved on the GRA [7/8(87.5\%) vs.

\footnotetext{
Corresponding Author: Yuko Komesu, MD, Department of Obstetrics and Gynecology, MSC 10-5580, 1 University of New Mexico, Albuquerque, NM 87131-0001, (505) 272-9712; Fax (505) 272-1336, ykomesu@ salud.unm.edu.

Authors contribution to Manuscript:

G. Kanter- Project development, patient recruitment, data analysis, manuscript writing

Y Komesu- Project development, patient recruitment, data analysis, manuscript editing

F Qaedan-Data analysis, manuscript editing

GC Dunivan- Project development, patient recruitment, manuscript editing

PC Jeppson- Project development, patient recruitment, manuscript editing

SB Cichowski- Project development, patient recruitment, manuscript editing

RG Rogers- Project development, patient recruitment, manuscript editing

Financial Disclosures/Conflicts of Interest:

G. Kanter-None

Y Komesu-None

F Qaedan-None

GC Dunivan-None

PC Jeppson-None

SB Cichowski-None

RG Rogers-DSMB Chair for the TRANSFORM trial sponsored by American Medical Systems, UptoDate royalties, Royalties from McGraw Hill for a textbook

${ }^{* *}$ This work is schedule to be presented as a full oral at the 42nd Annual Scientific Meeting of the Society of Gynecologic Surgeons in Palm Springs, CA scheduled for April 10-13, 2016, and will be published in the book of abstracts
} 
4/11(36.4\%), $\mathrm{p}=0.03]$. The MBSR group had greater improvement in OSPI total $(\mathrm{p}=0.0498)$ and problem scores $(\mathrm{p}=0.036)$; OSPI symptom score change did not differ. PSEQ scores improved in MBSR compared to UC ( $\mathrm{p}=0.035$ ). VAS, SF-12, and FSFI change did not differ between groups. Eighty-six percent of MBSR participants felt more empowered to control symptoms, and all participants planned to continue MBSR.

Conclusions-This trial provides initial evidence that MBSR is a promising adjunctive therapy for IC/BPS. Its benefit may arise from patients' empowerment and ability to cope with symptoms.

\section{MeSH Terms}

Chronic pain; Complementary Therapies; Cystitis; Interstitial; Emotional Stress; Mindfulness

\section{Introduction}

Interstitial cystitis/bladder pain syndrome (IC/BPS) is a chronic pain condition associated with significant morbidity. IC/BPS comprises a symptom complex defined by the American Urological Association (AUA) as "An unpleasant sensation (pain, pressure, discomfort) perceived to be related to the urinary bladder, associated with lower urinary tract symptoms of more than six weeks duration, in the absence of infection or other identifiable causes"[1]. Up to $11 \%$ of women are affected by IC/BPS [2], and the disorder may be significantly underdiagnosed [3]. Up to $43 \%$ of patients with IC/BPS require multimodal therapy with an average of 7-12 yearly clinic visits at a cost of $\$ 9,000 /$ patient per year.[4] Twenty percent of women report wage losses of $>\$ 4000$ per year [5]. The underlying pathophysiology of IB/BPS is poorly understood. Current theories include infectious agents, a defective glycosoaminoglycan layer of the urothelium which is permeable to toxic substances, structural abnormalities, neurogenic inflammation, increased neurologic sensitivity, or an allergic response involving increased numbers of bladder mast cells [6,7]. IB/BPS treatments involve trial and error of several therapies and often utilize multiple modalities $[1,8]$. Treatment success ranges from $47-93 \%$ for intravesical instillation to $21-64 \%$ for oral therapies, with discontinuation rates up to $80 \%$ due to undesirable side effects [1].

Mindfulness Based Stress Reduction (MBSR), a Complementary Alternative Medicine (CAM)-based therapy, is a standardized program including components of meditation and yoga. The Interstitial Cystitis Association, a patient-led organization, sponsored a survey of 2100 IC/BPS patients. They reported that $84 \%$ had tried CAM therapy and 55\% reported CAM therapy had been recommended by their physician[9]. According to a 2007 National Health Interview survey [10], CAM is used by $38 \%$ of adults in the United States. MBSR has been successfully employed to treat chronic pain syndromes and has been used in disorders such as multiple chemical sensitivity, chronic fatigue syndrome, fibromyalgia [11], various pelvic floor disorders[12] and irritable bowel syndrome (IBS) [13]. These disorders may coexist in patients with IC/BPS [6]. In IC/BPS, increased stress is positively correlated with increased pain [14] and up to $80 \%$ of IC/BPS patients noted in a survey that stress reduction decreased their symptoms [9]. MBSR has been reported to be efficacious in the treatment of urgency urinary incontinence, a urinary disorder closely related to IC/BPS [15]. Although MBSR has shown therapeutic benefit in pain disorders similar to IC/BPS and has been reported to be helpful by IC/BPS sufferers, data from randomized clinical trials 
investigating the efficacy of MBSR for IC are lacking [9]. The objective of this research was to provide initial data to evaluate MBSR as a treatment for patients with IC/BPS.

\section{Materials and Methods}

Patients with IC/BPS were recruited from the University of New Mexico (UNM) Urogynecology clinics. Participants were eligible to participate if they met AUA criteria for IC/BPS of "an unpleasant sensation (pain, pressure, discomfort) perceived to be related to the urinary bladder, associated with lower urinary tract symptoms of more than six weeks duration, in the absence of infection or other identifiable causes"[1] and had a minimum score of $\geq 8$ on the O'Leary-Sant Symptom Problem Index (OSPI). The O'leary-Sant symptom and problem index consists of symptom sub-score ranging from 0-21 as well as a problem sub-score ranging from 0-16 which evaluates how much bother IC/BPS symptoms cause, and a total score, which adds the symptoms and problem score (ranging from 0-37). Additional eligibility criteria included a negative urinalysis or urine culture within 2 months of enrollment and the ability to speak and understand English. We recruited only patients that were currently undergoing $1^{\text {st }}$ or $2^{\text {nd }}$ line treatments as defined by the AUA, and who had not made changes in their IC/BPS treatment regimen within 4 weeks of beginning their assigned intervention. First line treatments include relaxation/stress management, pain management and self-care/behavioral modification. Second line therapy involves physical therapy, as well as oral or intravesical medications. Patients who were treatment naïve or undergoing $3^{\text {rd }}$ line or higher treatments at the time of enrollment were excluded. Patients were also excluded if they had unevaluated hematuria, urinary retention, a history of cystectomy, augmentation cystoplasty or urinary diversion, a history of radiation or chemotherapy-induced cystitis, or if they were pregnant or lactating. All patients provided written informed consent and UNM Institutional Review Board approval was obtained prior to starting this study (HRRC \#14-141), which was registered in clinicaltrials.gov (NCT \#02457182).

This randomized controlled clinical trial compared 2 parallel treatment arms. Women were randomized to continue usual care (UC) or to an 8-week MBSR class plus continuation of their current care regimen. Both groups received standardized education including an educational handout. Participants were randomized at a 1:1 ratio by a computer generated sequence at enrollment. Allocation was concealed in serially-numbered opaque sealed envelopes, which were opened upon enrollment into the study. Participants were enrolled and randomized within 2 weeks prior to enrollment in a MBSR class cycle. Study participants and providers were not masked to intervention, however providers were asked not to make changes in IC/BPS treatment regimens during the study period unless specifically requested by study participants due to worsening of symptoms. Participants assigned to the MBSR intervention were enrolled in a local 8-week MBSR group course, taught by a certified MBSR instructor who had completed specialized MBSR training and had 12 years' experience teaching MBSR classes. The course was based upon MBSR workshops designed by Jon Kabat-Zinn [16,17], the recognized pioneer of this discipline. Participation in the $\$ 300$ MBSR course was paid for by study funding. The standardized course included seven 2-hr courses at weekly intervals with an all-day retreat in the $5^{\text {th }}$ week. These sessions taught meditation, yoga, and other relaxation techniques. In addition to 
the class room training, MBSR participants were given a 4-CD guide to meditation based on the prior work of Jon Kabat-Zinn and a book to assist with home meditation practice.

Our primary outcome was the improvement in the Global Response Assessment (GRA) between groups. GRA scores range from markedly, moderately or slightly worse to slightly, moderately or markedly improved. Our secondary outcomes included the OSPI(See above in methods for description), as well as a visual analog pain scale (VAS), the Short Form Health Survey (SF-12) which measures patient quality of life, the Female Sexual Function Index (FSFI) which consists of various domain scores and a total score, and the Pain Self-Efficacy Questionnaire (PSEQ). The PSEQ measures how one's coping ability is affected by pain. We powered our study to detect a 50\% difference in the proportion of individuals at least slightly improved between the MBSR and usual care groups. Assuming a significance level of $5 \%$ and a power of $80 \%$, a sample size of 20 women was needed to detect such a difference.

Demographic data, baseline questionnaires and clinical information including current and prior IC treatments were collected at enrollment. At the completion of the 8 week study period, both groups completed all study questionnaires. Patients completed forms in a nonclinical setting at the University Hospital. Continuous variables were analyzed using Student's t-test and categorical variables with the Chi-square test. Post-treatment GRA scores were compared between groups using the Chi-Square test. Multivariate Analysis of Variance (MANOVA) was used to compare changes in the other outcome measures (VAS, SF-12, Pain SEQ, FSFI) from baseline to post-treatment between groups, and its results were reported with the Wilks-Lambda test statistic. Significance was set at $\mathrm{P}<0.05$ and all analyses were carried out using SAS 9.4 statistical software (SAS Institute Inc., Cary, NC).

\section{Results}

Between September 2014 and April 2015 twenty patients were enrolled and randomized; 11 to UC, and 9 to MBSR (Figure I). One MBSR subject was lost to follow-up after randomization and did not attend any classes or answer follow-up surveys despite multiple contact attempts. All 8 patients who attended the MBSR class completed at least 50\% (4/8) of the classes, with 5 completing at least 75\% (6/8) of the classes (mean classes attended 5.9, SD 1.2). Patient characteristics did not vary significantly between groups with regards to age, race, socioeconomic status, duration of diagnosis of IC/BPS, previous treatments or obstetrical or surgical histories (Table I).

Compared to the UC group, more MBSR subjects rated their post-treatment symptoms based on the GRA to be improved (7/8 (87.5\%) vs. $4 / 11(36.4 \%), p=0.03)$. In addition, more women in the MSBR group reported markedly improved symptoms as compared to the UC group (2/8 (25\%) vs. $0 / 11(0 \%), p=0.08)$, though this was not statistically significant (Figure II). The MBSR group had greater improvement in OSPI total scores $(\mathrm{p}=0.05)$ and OSPI problem scores $(\mathrm{p}=0.04)$ (Figure III). However, changes in OSPI symptom scores did not differ significantly between the two groups $(\mathrm{p}=0.12)$. Patients' pain self-efficacy (PSEQ) scores also significantly improved in the MBSR group compared to the UC group ( $p$ 
$=0.04)$ (Table II). Changes in VAS scores, mental and physical components of the SF-12 quality of life score and FSFI scores did not differ between groups.

Eighty-six percent ( 6 of the 7 responders)) of patients in the MBSR group stated they felt more empowered to control their bladder symptoms following completion of the class than they did prior to attending the class. Five of 8 patients said they felt an improvement in their symptoms after a meditation session. Sixty-two percent (5/8) of the MBSR group indicated that they practiced home meditation after the course, with the same number noting improvement in symptoms after a meditation session. All 8 participants said they would continue to incorporate MBSR in their care plans for IB/BPS.

\section{Discussion}

Among women with a known diagnosis of IC/BPS, addition of MBSR to usual care resulted in greater global improvement in response to therapy (GRA) as well as OSPI total and problem scores and pain self-efficacy. Information regarding IC/BPS patients' experience with CAM and behavioral therapies has thus far been limited to surveys [9] and observational studies [18]. Our study provides initial evidence that participation in an 8week MBSR class results in improvement in IC/BPS compared to women using first or second line therapies from IC/BPS.

Given these improvements, it is possible that MBSR strategies provide coping mechanisms to deal with IC/BPS symptoms and pain. Researchers have suggested that the root cause of suffering in chronic pain may be attributable to associated helplessness and/or hopelessness. $[19,20]$ Perhaps the global improvement in symptoms in the MBSR group compared to controls was due to alleviation of some of the hopelessness associated with IC/BPS. The improved self-efficacy scores in the MBSR group suggest MBSR may diminish perceived helplessness associated with the chronic pain of IC/BPS. As opposed to acute pain, which is related to nociceptive signals at the level of tissue damage, chronic pain appears to be processed in regions of the brain associated with emotion and reward[21]. This may explain why therapies that target the bladder are incompletely effective in many patients. The addition of MBSR or other mind-body therapies to traditional treatments may enhance treatment response as the bladder may no longer be the sole mediator of symptoms in IC/ BPS. The known association of IC/BPS exacerbations with stress [14,22] supports the plausibility of MBSR as an adjunctive treatment for this condition.

Though MBSR has been used to successfully treat other chronic plain conditions such as fibromyalgia [23,24], irritable bowel syndrome [13] and multiple chemical sensitivity [11], use of MBSR to treat IC/BPS is understudied. In a cross-sectional survey[9], the majority of patients with IC/BPS rated both stress reduction and meditation as "helpful" treatment modalities. Our data provide strong initial evidence that MBSR may be a viable treatment for IC/BPS along with traditional $1^{\text {st }}$ and $2^{\text {nd }}$-line therapies. In contrast to existing treatments, MBSR is a patient-centered treatment which shifts care from time-consuming medical visits to self-initiated care. This empowers patients to address their condition with an intervention that they may be able to self-administer for long-term management. 
We note that quality of life and sexual function did not differ between groups after treatment. The lack of differences observed may have been due to the study's short followup period or small sample size. It is possible that improvement of pain, sexual function and quality of life would not be observed so soon after the intervention. We acknowledge that this study was an initial investigation into the feasibility and efficacy of MBSR therapy for IC/BPS and it establishes a need for future large studies with long-term follow-up to more definitively delineate the role that mind-body therapy may play in treatment of IC/BPS.

This study's strengths include its randomized controlled trial study design and its use of patient-centered, validated questionnaires as outcome measures. The MBSR intervention was selected as a standardized CAM treatment option that is increasingly offered throughout the country[25]. The reproducibility of the intervention adds to the study's external validity. Weaknesses of this study include its small sample size and short-term follow-up, though the large effect size in this smaller study does provide evidence upon which to power larger future work. In addition, this study used a control with a less active or involved intervention. This leaves open the possibility that the improved outcomes in the MBSR group may have been due to greater attention given to that group, and not something specific to the MBSR technique.

In conclusion, this study provides evidence to support the role of MBSR as a novel treatment for IC/BPS in patients undergoing concomitant $1^{\text {st }}$ and $2^{\text {nd }}$ line therapies. This treatment is a complementary modality that can be incorporated into the care plan of patients with IC/BPS, and should be considered as part of $1^{\text {st }}$ or $2^{\text {nd }}$ line therapy.

\section{Acknowledgments}

Thanks to Michelle DuVal for her support in this project and for providing MBSR teaching.

Registration: Clinicaltrials.gov: NCT02457182

Funding: This project was supported (in part or in full) by the National Center for Research Resources and the National Center for Advancing Translational Sciences of the National Institutes of Health through Grant Number UL1 TR001449. The content is solely the responsibility of the authors and does not necessarily represent the official views of the NIH.

\section{References}

1. Hanno PM, Burks DA, Clemens JQ, Dmochowski RR, Erickson D, Fitzgerald MP, Forrest JB, Gordon B, Gray M, Mayer RD, Newman D, Nyberg L Jr, Payne CK, Wesselmann U, Faraday MM. AUA guideline for the diagnosis and treatment of interstitial cystitis/bladder pain syndrome. The Journal of urology. 2011; 185(6):2162-2170. DOI: 10.1016/j.juro.2011.03.064 [PubMed: 21497847]

2. Rosenberg MT, Hazzard M. Prevalence of interstitial cystitis symptoms in women: a population based study in the primary care office. The Journal of urology. 2005; 174(6):2231-2234. DOI: 10.1097/01.ju.0000181203.82693.95 [PubMed: 16280776]

3. Clemens JQ, Meenan RT, O'Keeffe Rosetti MC, Brown SO, Gao SY, Calhoun EA. Prevalence of interstitial cystitis symptoms in a managed care population. The Journal of urology. 2005; 174(2): 576-580. [PubMed: 16006901]

4. Stanford EJ, Chen A, Wan GJ, Lunacsek OE, Sand PK. Treatment modalities, health care resource utilization, and costs in patients diagnosed with interstitial cystitis. American journal of obstetrics and gynecology. 2008; 199(1):71, e71-10. DOI: 10.1016/j.ajog.2008.02.048 [PubMed: 18585523] 
5. Clemens JQ, Markossian T, Calhoun EA. Comparison of economic impact of chronic prostatitis/ chronic pelvic pain syndrome and interstitial cystitis/painful bladder syndrome. Urology. 2009; 73(4):743-746. DOI: 10.1016/j.urology.2008.11.007 [PubMed: 19193408]

6. Dyer AJ, Twiss CO. Painful bladder syndrome: an update and review of current management strategies. Current urology reports. 2014; 15(2):384.doi: 10.1007/s11934-013-0384-z [PubMed: 24384999]

7. Logadottir Y, Delbro D, Lindholm C, Fall M, Peeker R. Inflammation characteristics in bladder pain syndrome ESSIC type 3C/classic interstitial cystitis. International journal of urology : official journal of the Japanese Urological Association. 2014; 21(Suppl 1):75-78. DOI: 10.1111/iju.12370 [PubMed: 24807505]

8. Cervigni M, Natale F, Nasta L, Padoa A, Voi RL, Porru D. A combined intravesical therapy with hyaluronic acid and chondroitin for refractory painful bladder syndrome/interstitial cystitis. International urogynecology journal and pelvic floor dysfunction. 2008; 19(7):943-947. DOI: 10.1007/s00192-008-0572-4 [PubMed: 18338095]

9. O'Hare PG 3rd, Hoffmann AR, Allen P, Gordon B, Salin L, Whitmore K. Interstitial cystitis patients' use and rating of complementary and alternative medicine therapies. International urogynecology journal. 2013; 24(6):977-982. DOI: 10.1007/s00192-012-1966-x [PubMed: 23149598]

10. Barnes PM, Bloom B, Nahin RL. Complementary and alternative medicine use among adults and children: United States, 2007. National health statistics reports. 2008; (12):1-23.

11. Sampalli T, Berlasso E, Fox R, Petter M. A controlled study of the effect of a mindfulness-based stress reduction technique in women with multiple chemical sensitivity, chronic fatigue syndrome, and fibromyalgia. Journal of multidisciplinary healthcare. 2009; 2:53-59. [PubMed: 21197347]

12. Slavin SL, Rogers RG, Komesu Y, Omotosho T, Hammil S, Lewis C, Sapien R. Complementary and alternative medicine (CAM) use in women with pelvic floor disorders: a cohort study. International urogynecology journal. 2010; 21(4):431-437. DOI: 10.1007/s00192-009-1058-8 [PubMed: 19967336]

13. Zernicke KA, Campbell TS, Blustein PK, Fung TS, Johnson JA, Bacon SL, Carlson LE. Mindfulness-based stress reduction for the treatment of irritable bowel syndrome symptoms: a randomized wait-list controlled trial. International journal of behavioral medicine. $2013 ; 20(3)$ : 385-396. DOI: 10.1007/s12529-012-9241-6 [PubMed: 22618308]

14. Rothrock NE, Lutgendorf SK, Kreder KJ, Ratliff T, Zimmerman B. Stress and symptoms in patients with interstitial cystitis: a life stress model. Urology. 2001; 57(3):422-427. [PubMed: 11248609]

15. Baker JNI, Costa D. Comparison of Mindfulness Based Stress Reduction (MBSR) vs Yoga on Urgency Incontinence: A Randomized Pilot Study. 2013 AUGS Abstract.

16. Kabat-Zinn J. An outpatient program in behavioral medicine for chronic pain patients based on the practice of mindfulness meditation: theoretical considerations and preliminary results. General hospital psychiatry. 1982; 4(1):33-47. [PubMed: 7042457]

17. Kabat-Zinn, J.; Hanh, TN. Full catastrophe living: Using the wisdom of your body and mind to face stress, pain, and illness. Random House LLC; 2009.

18. Whitmore KE. Complementary and alternative therapies as treatment approaches for interstitial cystitis. Reviews in urology. 2002; 4(Suppl 1):S28-35.

19. Ballantyne JC, Sullivan MD. Intensity of Chronic Pain--The Wrong Metric? The New England journal of medicine. 2015; 373(22):2098-2099. DOI: 10.1056/NEJMp1507136 [PubMed: 26605926]

20. Cassel EJ. The nature of suffering and the goals of medicine. The New England journal of medicine. 1982; 306(11):639-645. DOI: 10.1056/nejm198203183061104 [PubMed: 7057823]

21. Hashmi JA, Baliki MN, Huang L, Baria AT, Torbey S, Hermann KM, Schnitzer TJ, Apkarian AV. Shape shifting pain: chronification of back pain shifts brain representation from nociceptive to emotional circuits. Brain : a journal of neurology. 2013; 136(Pt 9):2751-2768. DOI: 10.1093/ brain/awt211 [PubMed: 23983029] 
22. Lutgendorf SK, Kreder KJ, Rothrock NE, Ratliff TL, Zimmerman B. A laboratory stress model for examining stress and symptomatology in interstitial cystitis patients. Urology. 2001; 57(6 Suppl 1): 122 .

23. Lauche R, Cramer H, Dobos G, Langhorst J, Schmidt S. A systematic review and meta-analysis of mindfulness-based stress reduction for the fibromyalgia syndrome. Journal of psychosomatic research. 2013; 75(6):500-510. DOI: 10.1016/j.jpsychores.2013.10.010 [PubMed: 24290038]

24. Merkes M. Mindfulness-based stress reduction for people with chronic diseases. Australian journal of primary health. 2010; 16(3):200-210. DOI: 10.1071/py09063 [PubMed: 20815988]

25. Pickert K. The Mindful Revolution. Time Magazine. Feb.2014 


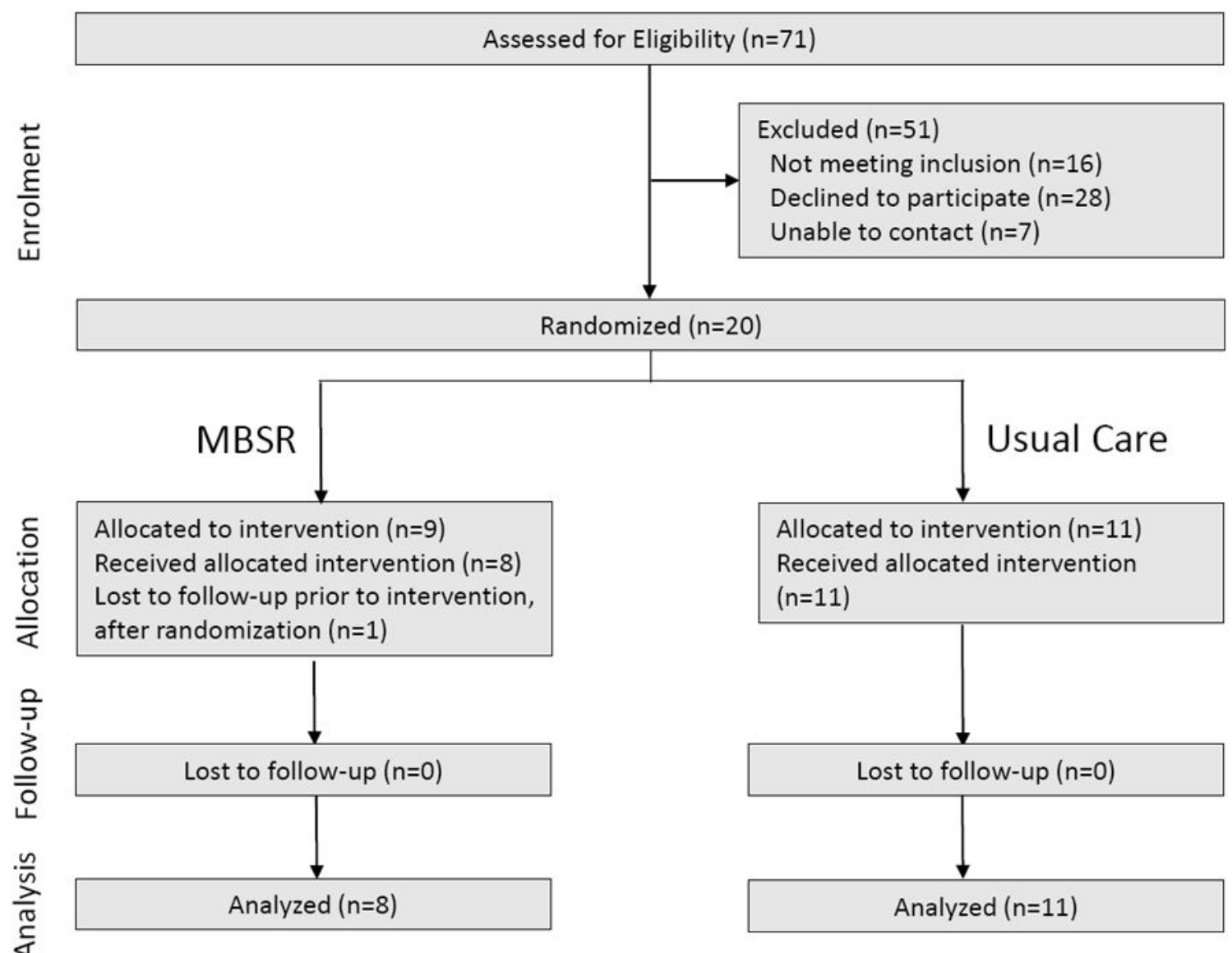

Figure I.

Consort diagram 
Global Response Assessment (GRA) following treatement

100

90

80

70

60

50

40

30

20

10

0

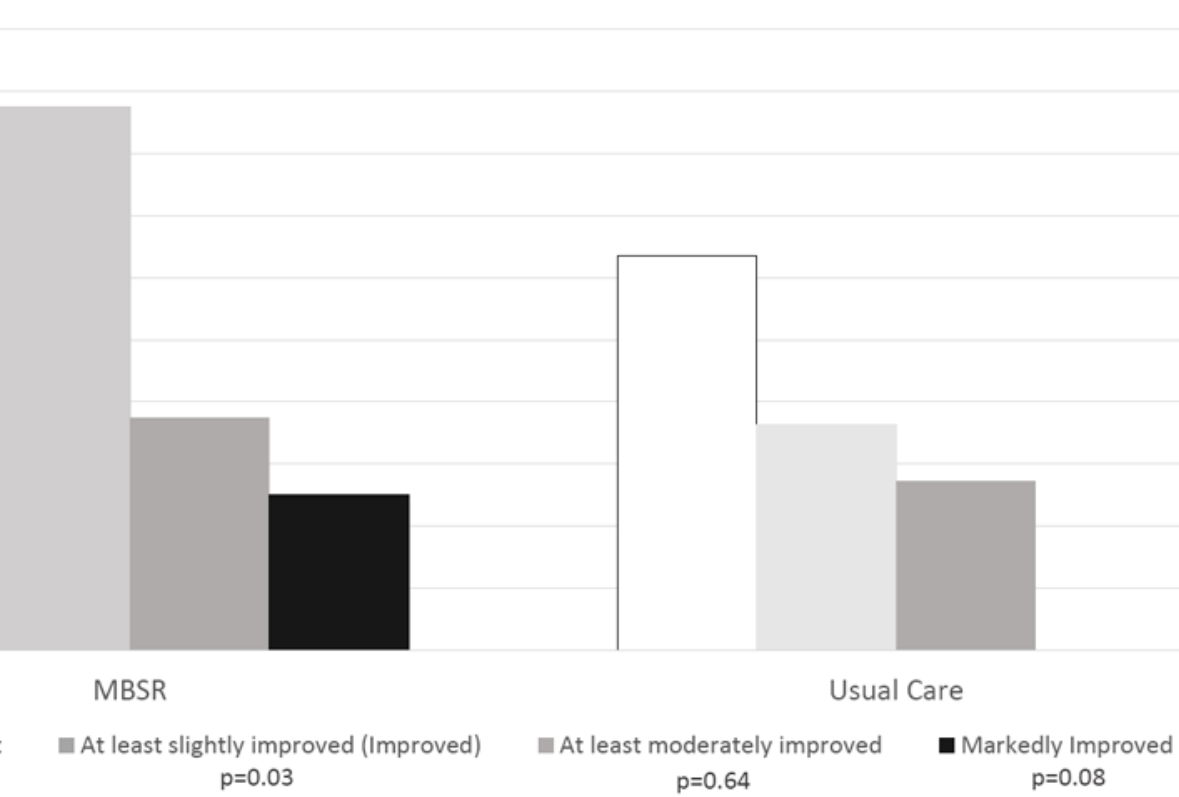

Figure II.

Global response assessment (GRA) after treatment. Reported p-values are obtained from the Chi-square test. 

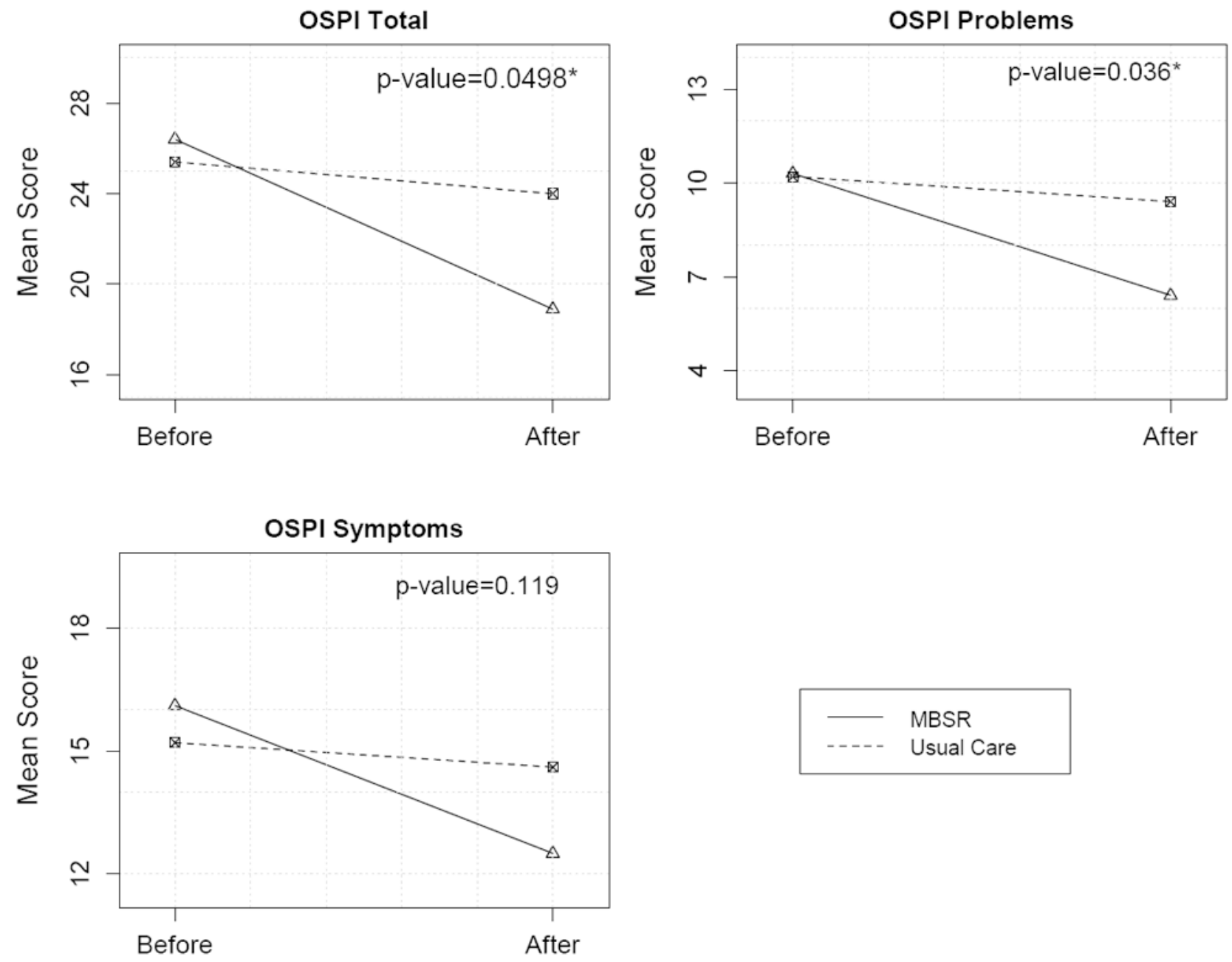

MBSR

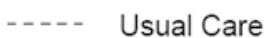

Figure III.

O'leary-Sant Total, Problem and Symptom mean scores before and after intervention for both intervention groups. Reported p-values are obtained from the MANOVA test. An asterisk (*) indicates the rejection of the null hypothesis at the 5 percent significance level (p-value less than 0.05 ). 


\section{Table 1}

Patient characteristics.

\begin{tabular}{|c|c|c|}
\hline Patient Characteristics & $\operatorname{MBSR}(\mathbf{n}=9)$ & Usual Care $(n=11)$ \\
\hline Age in years $(\mathrm{SD})$ & $46.3(15.2)$ & $44.4(13.9)$ \\
\hline Parity (SD) & $1.3(1.2)$ & $1.2(1.3)$ \\
\hline Vaginal deliveries (SD) & $1.3(1.2)$ & $0.8(1.1)$ \\
\hline Cesarean sections (SD) & 0 & $0.36(0.9)$ \\
\hline Time since IC Dx. in years (SD) & $5.0(5.3)$ & $3.85(5.1)$ \\
\hline Time with IC Sx. in years (SD) & $9.6(9.9)$ & $8.9(7.1)$ \\
\hline BMI $\left(\mathrm{kg} / \mathrm{m}^{2}\right)(\mathrm{SD})$ & $28.0(9.2)$ & $26.4(6.0)$ \\
\hline \multicolumn{3}{|l|}{ Surgical history (\%) } \\
\hline Incontinence & 0 & $2(18.2 \%)$ \\
\hline Prolapse & 0 & $1(9.1 \%)$ \\
\hline Hysterectomy & $3(33.3 \%)$ & $4(36.4 \%)$ \\
\hline \multicolumn{3}{|l|}{ POPQ Stage } \\
\hline 0 & $3(33.3 \%)$ & $2(18.2 \%)$ \\
\hline 1 & $4(44.4 \%)$ & $3(27.3 \%)$ \\
\hline 2 & $2(22.2 \%)$ & $6(54.6 \%)$ \\
\hline \multicolumn{3}{|l|}{ Race } \\
\hline American Indian/Alaskan Native & 0 & $2(18.8 \%)$ \\
\hline Asian & 0 & $1(9.1 \%)$ \\
\hline Caucasian & $9(100.0 \%)$ & $5(45.5 \%)$ \\
\hline Other & 0 & $3(27.3 \%)$ \\
\hline \multicolumn{3}{|l|}{ Insurance } \\
\hline Medicaid/Medicare & $1(11.1 \%)$ & $4(36.4 \%)$ \\
\hline Military & 0 & $2(18.2 \%)$ \\
\hline Multiple Insurances & $2(22.2 \%)$ & $1(9.1 \%)$ \\
\hline Private & $5(55.6 \%)$ & $4(36.4 \%)$ \\
\hline Self-pay & $1(11.1 \%)$ & 0 \\
\hline \multicolumn{3}{|l|}{ Education } \\
\hline High school or equiv. & 0 & $2(18.2 \%)$ \\
\hline Some college & $2(22.2 \%)$ & $4(36.4 \%)$ \\
\hline Associate's Degree & $1(11.1 \%)$ & $1(9.1 \%)$ \\
\hline Bachelor's or graduate Degree & $6(66.7 \%)$ & $4(36.4 \%)$ \\
\hline \multicolumn{3}{|l|}{ Previous Treatments Tried } \\
\hline Physical Therapy & $4(44.4 \%)$ & $5(45.5 \%)$ \\
\hline
\end{tabular}

Int Urogynecol J. Author manuscript; available in PMC 2017 November 01. 


\begin{tabular}{|r|l|l|}
\hline Patient Characteristics & MBSR (n=9) & Usual Care (n=11) \\
\hline Oral Medications & $8(88.9 \%)$ & $9(81.8 \%)$ \\
Elimination Diet & $6(66.7 \%)$ & $6(54.6 \%)$ \\
\hline Number of previous medications tried & 7 respondents & 8 respondents \\
1 & $1(14.3 \%)$ & $3(37.5 \%)$ \\
2 & $3(42.9 \%)$ & $1(12.5 \%)$ \\
3 & 0 & $2(25.0 \%)$ \\
4 or more & $3(42.9 \%)$ & $2(25.0 \%)$ \\
\hline Current Treatment & & \\
Bladder instillations & $1(11.1 \%)$ & $3(27.3 \%)$ \\
Physical therapy & $1(11.1 \%)$ & $1(9.1 \%)$ \\
\hline
\end{tabular}

Continuous variables are describes by the sample mean and standard deviation (SD), categorical variables are describes by the frequency and relative frequency (\%).No significant differences were noted between groups; $p>0.05$ for all $t$-tests and Chi-square tests. $\mathrm{POPQ}=$ pelvic organ prolapse quantification. 
